\title{
Entornos Personales de Aprendizaje para la comprensión y desarrollo de la Competencia Digital: análisis de los estudiantes universitarios en España
}

\section{Personal Learning Environments to understand and develop Digital Competence: analysis of University students in Spain}

\author{
M. $^{\text {a Paz Prendes Espinosa }}{ }^{1}$ \\ pazprend@um.es \\ Isabel María Solano Fernández \\ imsolano@um.es \\ José Luis Serrano Sánchez \\ jl.serranosanchez@um.es \\ Víctor González Calatayud \\ victor.gonzalez@um.es \\ M. a del Mar Román García \\ mariamar.roman@um.es \\ Universidad de Murcia, España
}

\section{Resumen:}

Tanto en el contexto internacional como nacional, se observa que cada vez es más importante la concreción de las competencias digitales en el contexto educativo, referidas tanto a los docentes como a los discentes. Si nos fijamos de modo más específico en los estudiantes, la competencia digital es un amplio constructo teórico que entre sus dimensiones sustenta la necesidad de analizar y potenciar el Entorno Personal de Aprendizaje (PLE), constructo

\begin{abstract}
:
Both at international and national levels it is observed that it is increasingly important to specify digital competences in the educational context, referring both to teachers and students. If we look more specifically at students, digital competence is a broad theoretical construct that, among its dimensions, supports the need to analyze and enhance the Personal Learning Environment (PLE), a construct that supports the CAPPLE Project and that allows us to
\end{abstract}

1 Dirección para correspondencia (correspondence address):

M. ${ }^{a}$ Paz Prendes Espinosa. Dpto. de Didáctica y Organización Escolar. Universidad de Murcia. Facultad de Educación. Campus de Espinardo, s/n. 30100 Murcia (España). 
Entornos Personales de Aprendizaje para la comprensión y desarrollo de la Competencia Digital: análisis de los estudiantes universitarios en España

M. ${ }^{a}$ Paz Prendes Espinosa, Isabel María Solano Fernández, José Luis Serrano Sánchez, Víctor González Calatayud y M. a del Mar Román García

que sustenta el Proyecto CAPPLE y que nos permite comprender de modo global cómo los estudiantes utilizan su competencia digital en sus procesos académicos de aprendizaje. En este artículo mostramos los principales resultados del Proyecto CAPPLE, un estudio exploratorio en el que se ha utilizado un cuestionario ad hoc para determinar las estrategias y herramientas tecnológicas que los estudiantes universitarios de último curso $(n=2054)$ utilizan en los procesos de aprendizaje y comunicación. El cuestionario ha sido validado con un triple procedimiento y un alpha de Cronbach de 0.944. Las evidencias obtenidas nos permiten desmitificar el concepto de nativo digital, puesto que los estudiantes de último curso de grado han integrado las tecnologías para comunicarse en sus espacios personales y de ocio, pero no tanto para su trabajo académico en la universidad.

\section{Palabras clave:}

Competencia digital; PLE; aprendizaje; comunicación; investigación; tecnología educativa. understand in a global way how students use their digital competence in their academic learning processes. In this paper we show the main results of the CAPPLE Project, an exploratory study in which an ad hoc questionnaire was used to determine the strategies and technological tools that final-year university students $(n=2,054)$ use in their learning processes and communication. The questionnaire was validated with a triple procedure and a Cronbach's alpha of 0,944. The evidence obtained allows us to demystify the concept of the "digital native", since senior students integrated technologies to communicate in their personal and leisure spaces, but not too much in their academic activities.

\section{Keywords:}

Digital competence; PLE; learning; communication; research; educational technology.

\section{Resumé:}

Qu'il s'agisse d'un contexte international ou national, on observe que la concrétisation et l'usage des compétences numériques dans le milieu éducatif sont de plus en plus importants aussi bien pour le corps enseignant que pour le corps étudiant. Si nous nous centrons maintenant sur les étudiants, la compétence numérique est une vaste construction théorique qui, dans toutes ses dimensions, assure la nécessité d'analyser et de stimuler I'Environnement Personnel d'Apprentissage (PLE). Une construction qui poursuit le projet CAPPEL et qui nous permet de comprendre de manière générale, comment les étudiants utilisent cette compétence dans leurs processus académiques d'apprentissage. Dans cet article, nous exposons les principaux résultats du Projet CAPPLE. Il s'agit d'une étude exploratoire dans laquelle nous avons utilisé un questionnaire ad hoc, afin de déterminer les stratégies et les outils technologiques que les étudiants universitaires en dernière année $(n=2054)$, utilisent dans leurs processus d'apprentissage et de communication. Ce questionnaire a été validé selon un triple critère ainsi qu'un alpha de Cronbach de 0.944. Les évidences obtenues nous ont permis de démystifier le concept de natif numérique, puisque les étudiants en dernière année de licence, ont surtout intégré les nouvelles technologies pour communiquer dans leurs espaces personnels et pendant leurs loisirs. La compétence numérique semble ne pas être autant utilisée leur travail de formation universitaire.

\section{Mots clés:}

Compétence numérique; PLE; apprentissage; communication; recherche; technologie éducative.

Fecha de recepción: 12-2-2018

Fecha de aceptación: 25-4-2018 
Entornos Personales de Aprendizaje para la comprensión y desarrollo de la

Competencia Digital: análisis de los estudiantes universitarios en España

M. a Paz Prendes Espinosa, Isabel María Solano Fernández, José Luis Serrano Sánchez, Víctor González Calatayud y M. ${ }^{a}$ del Mar Román García

\section{Investigación en tecnología educativa}

La Tecnología Educativa (TE), en tanto que disciplina propia del área de Didáctica y Organización Escolar, ha evolucionado considerablemente a lo largo de los años. Desde su constitución como disciplina se ha ido pasando de una visión instrumental a una visión más instructiva de los medios, centrada en el análisis y diseño del proceso para la consecución de objetivos de aprendizaje. También se ha hecho hincapié en la necesidad de realizar un análisis del contexto y los aspectos sociales que rodean a las tecnologías (García-Valcárcel, 2003; Prendes y Serrano, 2016; Sancho, 1998).

Escudero (1995) en un magnífico análisis sobre la relación existente entre TE, reestructuración escolar e innovación, ha sido un autor de referencia en relación con las formas de abordar la TE e identifica dos perspectivas claramente diferenciadas: la TE como eje nuclear de la transformaciones y reformas escolares y educativas, y la TE en el centro de un movimiento de contestación y resistencia que aboga por "un cambio de paradigma de porte más humano cultural, antropológico y emancipador como respuesta al tecno-científico" (p.168). El autor apuntaba desde una perspectiva crítica, la imposibilidad de concebir el objeto y proceso que estudia la TE como neutral:

La Tecnología educativa merece ser entendida como una mirada y un conjunto de procesos y procedimientos, no solo aparatos (1), con vocación de conformar tanto un modo de pensar la educación como una línea operativa de ordenación y actuación en este ámbito, Ilevando asociada, por tanto, relaciones entre los sujetos y aquellos que detentan el poder político, económico y organizativo para su diseño, desarrollo y control. (Escudero, 1995, p. 162).

El análisis de la investigación en TE ha puesto de manifiesto una enorme evolución (Cabero, 2016; De Benito y Salinas, 2016), encontrándose una importante producción en revistas especializadas y no especializadas del ámbito de la TE (Valverde, 2016).

Cabero (2003) explicó esta evolución haciendo referencia al paso de una perspectiva micro, centrada en los artefactos, a una perspectiva más global. El autor apunta que el replanteamiento de la TE pasa inevitablemente por centrarse en el "diseño, la utilización y la evaluación de 
las tecnologías de la información aplicadas a acciones formativas, sean éstas formales, informales o no formales (...) este cuerpo de conocimientos implica el juicio crítico de las TIC no sólo en cuanto instrumento tecnológico, sino también como sistemas de representación, de análisis de la realidad circundante y de herramientas cognitivas de apoyo a la persona" (p. 26).

Para Cabero (2016), la investigación en TE se ha movido en dos grandes visiones: la aplicación de los medios y el diseño de la instrucción. En la misma línea, Prendes y Serrano (2016) y Serrano, Gutiérrez y Prendes (2016) apuntan que en la investigación en TE ha sido frecuente confundir el uso de tecnologías como herramientas de carácter transversal y de propósito múltiple con el uso educativo de tecnologías y la investigación sobre ello.

En el análisis que realiza Valverde (2016) sobre la investigación en TE, destaca los estudios realizados por Baydas, Kucuk, Yilmaz, Aydemir y Goktas (2015) y Hsu, Hung y Ching (2013). Para Baydas et al. (2015) los principales núcleos de interés de la TE son las teorías y enfoques de aprendizaje, los entornos de aprendizaje, el aprendizaje online, los estudios sobre diseño curricular y los estudios de evaluación. Por otra parte, el meta-análisis realizado por Hsu et al. (2013) durante el período 2000-2010, a partir de artículos publicados en seis revistas especializadas en TE, destaca tres grandes categorías como temas principales de la investigación en este ámbito: la macrovisión del e-learning e integración de las Tecnologías, aceptación/actitud hacía las tecnologías emergentes y los entornos de aprendizaje.

Por su parte, Cabero (2016, p. 31) apunta las diferentes líneas y problemáticas de investigación en un futuro en TE:

- Estrategias de formación del profesorado para el uso técnico, sémico y didáctico de los medios.

- Utilización de las TIC para sujetos con necesidades educativas especiales.

- Interacción que el sujeto establece con los nuevos canales de comunicación y con la web 2.0.

- Utilización de los medios para la creación de contextos significativos de aprendizaje (aprendizaje cooperativo, aprendizaje situado, aprendizaje aumentativo...).

- Análisis de la dimensión organizativa para la introducción de las TIC. 
Entornos Personales de Aprendizaje para la comprensión y desarrollo de la

Competencia Digital: análisis de los estudiantes universitarios en España

M. a Paz Prendes Espinosa, Isabel María Solano Fernández, José Luis Serrano Sánchez, Víctor González Calatayud y M. ${ }^{a}$ del Mar Román García

- Transferencia de información vía medio.

- Realización de entornos de telemáticos de formación flexibles y bajo el concepto "just- in-time".

- Estudios sobre la significación social de las tecnologías. Brecha digital.

- Currículum oculto en las nuevas tecnologías.

- Valores que transmiten las TIC.

- Análisis de la dimensión organizativa para la introducción de las TIC.

- Estudios económicos preocupados en el análisis coste-beneficio de incorporación de las TIC.

- Género e interacción con TIC (Tecnofeminismo).

- La construcción social del conocimiento a través de las TIC (Redes sociales).

- Impactos sociales, culturales y psicológicos de los medios.

- Analizar las posibilidades educativas de nuevas tecnologías como por ejemplo la web semántica, los entornos personales de aprendizaje, la realidad aumentada, o la gamificación.

En un reciente trabajo sobre los procesos de innovación educativa, Escudero (2014) reconoce de forma explícita su vinculación con la Tecnología Educativa. De entre las lecciones que enuncia para el logro de una auténtica renovación de las aulas, los centros y el sistema en su conjunto, destacamos la que hace referencia a la necesidad de poner nuestra mirada en la mejora del currículum escolar con acciones en las que las tecnologías tendrían un papel clave.

Y hablar de TE en la Universidad de Murcia supone hablar del trabajo del Grupo de Investigación de Tecnología Educativa² (GITE) creado por Francisco Martínez Sánchez en el contexto del Departamento de Didáctica y Organización Escolar que dirigía como Catedrático Juan Manuel Escudero Muñoz. Desde entonces hasta hoy, el GITE ha evolucionado y ha crecido hasta llegar al momento actual, siendo por su producción científica y su impacto, uno de los más relevantes de la universidad española.

Al igual que ha ido evolucionando la TE en estas décadas, como se ha podido ver, también los intereses del GITE han ido cambiando. Mientras en los años 90 nuestra investigación se centraba en los libros de texto,

2 Web: http://www.um.es/gite/ 
el vídeo, las imágenes didácticas o el software educativo, en esta última década nuestros intereses se han dirigido hacia la formación en línea, los OER, los MOOC, el PLE, la competencia digital y la innovación educativa en contextos como las aulas hospitalarias. $Y$ es necesario reconocer que la TE se ha convertido en un ámbito de conocimiento de enorme relevancia, dada la cada vez mayor interrelación de la innovación tecnológica y la innovación de la enseñanza universitaria en un contexto social cada vez también más cambiante.

Las tecnologías forman parte de la realidad que nos ha tocado vivir y en opinión de Marcovitch (2002, p. 87):

Ante la revolución tecnológica, la universidad se comporta como cualquier otra organización de nuestro tiempo. No puede ignorarla y dejar de aprovechar todos sus beneficios. Evidentemente, como centro crítico y cuestionador por naturaleza, la universidad jamás será una usuaria incondicional de las oportunidades creadas por la tecnología, pero desconocerla o dejar de aprovecharla, cuando se hace necesario, es absolutamente imperdonable.

En este contexto es de especial interés la investigación sobre la competencia digital del profesorado y la competencia digital del alumnado, investigación cuyos resultados presentamos en este artículo.

\section{Competencia digital}

Monereo (2005, p.13) entiende que "alguien competente es una persona que sabe leer con gran exactitud qué tipo de problema es el que se le plantea y cuáles son las estrategias que deberá activar para resolverlo". Muy conocida es la aproximación de Perrenoud (2004, p.11), quien define que la "competencia es la capacidad de movilizar varios recursos cognitivos para hacer frente a un tipo de situaciones". Siguiendo estas definiciones, un profesional competente ha de "ser capaz de transferir y adaptar, en el marco de su desempeño laboral, uno o varios esquemas de actividad a diversas situaciones o problemas" (Pavié, 2011, p.79).

A partir de este concepto de competencia, la Comisión Europea (2006) concreta 8 competencias clave del ciudadano del siglo XXI: comunicación en la lengua materna; competencia en matemáticas, ciencia y tecnología; competencia digital; aprender a aprender; social y cívica; 
Entornos Personales de Aprendizaje para la comprensión y desarrollo de la Competencia Digital: análisis de los estudiantes universitarios en España

M. a Paz Prendes Espinosa, Isabel María Solano Fernández, José Luis Serrano Sánchez, Víctor González Calatayud y M. ${ }^{a}$ del Mar Román García

emprendimiento; expresión y conciencia cultural. Y si la competencia digital es básica desde la perspectiva de la ciudadanía, por ende hemos de concebirla como una competencia básica de alumnado y profesorado en los modelos de formación del siglo XXI. Se entiende la competencia digital como:

La competencia digital entraña el uso seguro y crítico de las tecnologías de la sociedad de la información (TSI) para el trabajo, el ocio y la comunicación. Se sustenta en las competencias básicas en materia de TSI: el uso de ordenadores para obtener, evaluar, almacenar, producir, presentar e intercambiar información, y comunicarse y participar en redes de colaboración a través de Internet" (Comisión Europea, 2006, p.15).

Gutiérrez (2014) aglutina las diversas dimensiones de la competencia digital al definirla como los "valores, creencias, conocimientos, capacidades y actitudes para utilizar adecuadamente las tecnologías, incluyendo tanto los ordenadores como los diferentes programas e Internet, que permiten y posibilitan la búsqueda, el acceso, la organización y la utilización de la información con el fin de construir conocimiento" (p.54).

En 2006, la LOE (Ley Orgánica 2/2006, de mayo, de Educación) incorpora en el contexto español la importancia de la competencia digital, entendiéndola como las habilidades para transformar la información en conocimiento. Con posterioridad la LOMCE (Ley Orgánica 8/2014, de 9 de diciembre, para la mejora de la calidad educativa) también recoge la competencia digital como una de las 7 competencias clave para la formación del alumnado y afirma que las TIC son elementos básicos para la innovación metodológica y la mejora de la calidad de la enseñanza. Posteriormente, la Comisión Europea elabora el "Marco para el Desarrollo y el Conocimiento de la Competencia Digital en Europa" (Ferrari, 2013), modelo que fue posteriormente revisado en 2016 y que ha sido utilizado por el INTEF para elaborar su propio modelo de Competencia Digital Docente.

En el contexto específico de la educación, podemos referirnos a la Competencia Digital de modo diferenciado según hablemos de las competencias digitales de los profesores (Competencia Digital Docente) o bien si nos referimos a las competencias digitales del alumnado, siendo en este caso un concepto directamente ligado al de Entorno Personal de Aprendizaje (véase la relación conceptual entre ambos en Suárez y Serrano (2016). 
En primer lugar, sobre la competencia digital del profesorado hemos de señalar la importancia de analizarla en relación con las competencias profesionales. En palabras de Tejada y Navío (2005, p.5), "la utilidad de la competencia profesional está en la capacidad de ésta para hacer frente a contextos profesionales cambiantes y en los que aspectos como la polivalencia y la flexibilidad son necesarios" y se puede adquirir y desarrollar gracias a la formación inicial, a la formación continua y a la propia actividad profesional. No vamos a hacer un análisis exhaustivo del concepto de competencia docente, pues excede el objetivo de este artículo, pero sí nos parece interesante recoger la definición de Escudero (2006, p. 34), quien concreta diversas dimensiones (Tabla 1) basándose en su propia definición de competencia docente entendida como:

Conjunto de valores, creencias y compromisos, conocimientos, capacidades y actitudes que los docentes, tanto a título personal como colectivo (formando parte de grupos de trabajo e instituciones educativas) habrían de adquirir y en las que crecer para aportar su cuota de responsabilidad para garantizar una buena educación a todos.

Tabla 1. Competencia docente (basado en Escudero, 2006).

\begin{tabular}{cl}
\hline $\begin{array}{c}\text { Conocimiento de base } \\
\text { sobre: }\end{array}$ & - Aprendizaje y diversidad del alumnado \\
& - Metodologías y estrategias \\
\hline & - Planificar la enseñanza \\
& - Seleccionar y crear tareas \\
Capacidades de & - Crear y mantener el clima de convivencia \\
aplicación del & - Crear situaciones instructivas \\
conocimiento para: & - Usar estrategias de comunicación verbal y no verbal \\
& - Usar variedad de estrategias instructivas \\
& - Evaluación \\
\hline & - Realizar prácticas éticas y compartiendo responsa- \\
Responsabilidad & bilidades \\
profesional para: & - Reflexión y aprendizaje continuo \\
& - Liderazgo y colaboración \\
\hline
\end{tabular}

Como definición de competencia digital docente recogemos la de Carrera y Coiduras (2012, p.15) para quienes este concepto incluye:

conocimientos, capacidades, actitudes y estrategias que (...) el profesor o el educador debe ser capaz de activar, adoptar y gestionar en situaciones 
Entornos Personales de Aprendizaje para la comprensión y desarrollo de la Competencia Digital: análisis de los estudiantes universitarios en España

M. a Paz Prendes Espinosa, Isabel María Solano Fernández, José Luis Serrano Sánchez, Víctor González Calatayud y M. ${ }^{a}$ del Mar Román García

reales para facilitar el aprendizaje alcanzando mayores niveles de logro y promover procesos de mejora e innovación permanente en los procesos formativos.

Quizás uno de los modelos de competencia docente más citado en los últimos años es el de Mishra y Koehler (2006), el modelo TPACK (Technological Pedagogical Content Knowledge) sobre el conocimiento tecnológico, pedagógico y disciplinar. Las interacciones de estos tres tipos de competencia acaban configurando el modelo ideal de docente competente en los tres ámbitos. Un instrumento de evaluación de la competencia según el modelo TPACK puede encontrarse en Cabero y Barroso (2016) y en Cabero, Marín y Castaño (2016). Vemos pues que todos estos autores destacan la relevancia del conocimiento de las tecnologías como una dimensión fundamental de la competencia docente. En relación con ello, en Durán, Gutiérrez y Prendes (2016) podemos encontrar una revisión sistemática de los diversos modelos de competencia digital docente y una propuesta de modelo centrada en el nivel universitario.

En segundo lugar nos referiremos a la competencia digital del alumnado, concepto que aparece relacionado con las diversas líneas de investigación sobre educación mediática, alfabetización digital, alfabetización mediática,... y también la investigación sobre el de Entorno Personal de Aprendizaje (o PLE). En los trabajos sobre competencia digital del alumnado se parte de la idea de que es ésta una competencia necesaria y relevante en la formación del ciudadano del siglo XXI, como ya hemos señalado, pues es una competencia necesaria además para afrontar el aprendizaje a lo largo de la vida. Sobre este tema es relevante citar el trabajo realizado en el marco del proyecto DeSeCo, en el cual se determinan las competencias clave para el ciudadano del siglo XXI. El ejemplo de sistema educativo donde quizá más se trabaja la competencia digital es el escandinavo, según Ferrari (2013), que ha sido bien descrito y analizado por Krumsvik (2008).

En España, tanto en la Ley Orgánica de Educación (RD 2/2006) en su Artículo 17 referido a los objetivos de la Educación Primaria, como posteriormente el Real Decreto 126/2014 que establece el currículum básico de la Educación Primaria (Artículo 2.2), se considera la competencia digital como uno de los pilares de la formación del alumnado junto con las otras 6 competencias clave para los ciudadanos del siglo XXI. Y 
también en la LOE, en los Artículos 23 y 33 referidos a la Enseñanza Secundaria Obligatoria, se señala como objetivo la adquisición de competencias para el uso de tecnologías de la información y la comunicación, lo que redunda en este ámbito que estamos analizando.

Así pues, nuestro enfoque de análisis de la competencia digital se asienta en su relación con el análisis de los Entornos Personales de Aprendizaje en tanto que constructo que nos permite estudiar y comprender las competencias para el aprendizaje apoyado en tecnologías (aprendizaje enriquecido con tecnologías o "technology enhanced learning"). Vamos a ahondar en la investigación sobre PLE del alumnado universitario en el siguiente apartado.

\section{Investigación sobre ple del alumnado universitario en España}

\subsection{Proyecto CAPPLE}

El proyecto CAPPLE$^{3}$ (Competencias para el aprendizaje permanente basado en el uso de PLE -Entornos Personales de Aprendizaje-: análisis de los futuros profesionales y propuestas de mejora) es un proyecto de investigación coordinado por $\mathrm{M}^{\mathrm{a}}$ Paz Prendes en el que han participado un total de 34 investigadores procedentes de 6 universidades diferentes. Además, se ha contado con el asesoramiento de investigadores internacionales. Este estudio (2013-2017) ha sido financiado por el Ministerio de Economía y Competitividad (España) dentro del "Subprograma de proyectos de investigación fundamental no orientada», en el marco del VI Plan Nacional de Investigación Científica, Desarrollo e innovación Tecnológica (Referencia EDU2012-33256).

La investigación se llevó a cabo desde un diseño no experimental, de tipo exploratorio y basado en el uso de un cuestionario como instrumento de recogida de información. El principal objetivo del proyecto CAPPLE es describir y analizar las estrategias y herramientas concretas utilizadas habitualmente por estudiantes universitarios de último curso (de 75 universidades diferentes) de todas las ramas de conocimiento para enriquecer su proceso de aprendizaje, dentro y fuera de las aulas, especialmente aquellas que tienen lugar en contextos electrónicos. Este análisis se apoya en la idea de que un PLE enriquecido supondrá un

3 Web: http://www.um.es/ple/_ 
pilar que sustente la competencia para el aprendizaje permanente de los estudiantes universitarios una vez que concluyan sus estudios y accedan al mercado laboral como profesionales. Desde esta perspectiva y con la evidencias que mostramos en este artículo, consideramos que conceptualmente el PLE está de modo intrínseco ligado al concepto de competencia digital.

Al contar con una población de estudio amplia y geográficamente dispersa (298000 estudiantes) se optó por una técnica de difusión a los participantes elegidos de modo intencional. Por lo tanto, no contamos con una muestra representativa de la población sino que hemos buscado que la muestra (tabla 2) sea amplia ( $\mathrm{n}=2054$ estudiantes) para poder obtener esos patrones que nos permitan encontrar perfiles personales en relación con el concepto de PLE. Desde el principio del proyecto se tuvo en cuenta que los estudios exploratorios no constituyen un fin en sí mismo, sino que nos ayudarían a determinar tendencias y relaciones entre variables además de, ofrecernos la posibilidad de delimitar posibles líneas futuras de investigación. La principal característica de este tipo de estudios es su flexibilidad en comparación con los descriptivos, correlacionales o explicativos (Hernández, Fernández-Collado y Baptista, 2006, p. 102).

Tabla 2. Población y muestra.

\begin{tabular}{ccccc}
\hline $\begin{array}{c}\text { Área de } \\
\text { Conocimiento }\end{array}$ & $\begin{array}{c}\text { Estudiantes de } \\
\text { último curso }\end{array}$ & $\begin{array}{c}\text { Porcentaje de estudian- } \\
\text { tes de último curso }\end{array}$ & Muestra & $\begin{array}{c}\text { Muestra } \\
(\%)\end{array}$ \\
\hline $\begin{array}{c}\text { Ciencias Sociales } \\
\text { y Jurídicas }\end{array}$ & 153952 & 52.2 & 879 & 42.79 \\
\hline $\begin{array}{c}\text { Ingeniería y } \\
\text { Arquitectura }\end{array}$ & 78265 & 26.4 & 277 & 13.49 \\
\hline $\begin{array}{c}\text { Artes y } \\
\text { Humanidades }\end{array}$ & 19966 & 6.8 & 308 & 15 \\
\hline $\begin{array}{c}\text { Ciencias de la } \\
\text { Salud }\end{array}$ & 29844 & 10.1 & 352 & 17.14 \\
\hline Ciencias & 16044 & 5.4 & 238 & 11.58 \\
\hline Total & 298071 & 100 & 2054 & 100 \\
\hline
\end{tabular}

Uno de los principales hitos logrados en esta investigación ha sido el diseño y la validación de un cuestionario. Tanto la versión final del instrumento como el proceso seguido en su elaboración y validación (triple procedimiento) se encuentran recogidos en Prendes et al. (2016), el cual podemos resumirlo de la siguiente manera: 
- Etapa 1: a raíz de la revisión teórica se construye un modelo de PLE articulado en 4 dimensiones (autopercepción, gestión de la información, gestión del proceso de aprendizaje y comunicación).

- Etapa 2: mediante el procedimiento de juicio de expertos, se llevó a acabo una segunda validación del contenido del cuestionario.

- Etapa 3: con el cuestionario modificado tras el juicio de expertos, se realizaron entrevistas cognitivas con 24 estudiantes de último curso de Grado de 8 universidades españolas diferentes y de las 5 ramas de conocimiento.

- Etapa 4: finalmente se realizó un estudio piloto con 400 estudiantes universitarios (268 mujeres, 132 hombres) de último curso de Grado.

Este instrumento tiene una fiabilidad de 0.944 en la prueba de Alfa de Cronbach y está compuesto por 48 preguntas cerradas (con 230 ítems). A pesar de su longitud, considerando su carácter de investigación exploratoria y nuestro afán por obtener datos descriptivos de la compleja realidad estudiada (Stake, 2010), optamos por no reducirlo.

Mediante el uso de una técnica de difusión a los participantes elegidos de modo intencional, se obtuvieron un total de 4399 encuestas, siendo la muestra final de nuestro proyecto 2054 casos completos, es decir, aquellos estudiantes que respondieron al cuestionario completo.

\subsection{Análisis de datos y resultados}

En coherencia con los objetivos, el diseño de la investigación y la muestra participante, se ha llevado a cabo un análisis descriptivo de cada variable categórica. Hemos reagrupado los valores de las respuestas en tres categorías: valores relativos a opciones de respuesta positivos (totalmente de acuerdo y de acuerdo), valores relacionados con opciones de respuesta neutrales y valores sobre opciones de respuesta negativos (totalmente en desacuerdo y en desacuerdo).

En el proceso de interpretación de los datos realizamos diferentes grupos de trabajo (técnica de grupos focales). En esta tarea participaron un total de 26 expertos divididos en tres grupos que trabajaron bajo estas tres ideas marco:

- Identificar principales rasgos y características relacionadas con el aprendizaje. 
Entornos Personales de Aprendizaje para la comprensión y desarrollo de la Competencia Digital: análisis de los estudiantes universitarios en España M. a Paz Prendes Espinosa, Isabel María Solano Fernández, José Luis Serrano Sánchez, Víctor González Calatayud y M. ${ }^{a}$ del Mar Román García

- Identificar posibles temas de investigación emergentes.

- Poner de relieve cuestiones que deben tenerse en cuenta antes de continuar análisis más profundos.

Tras realizar este análisis de datos, en una siguiente fase se dividió la base de datos en dos partes equivalentes con las cuales se ha replicado el análisis factorial exploratorio y se ha realizado un análisis factorial confirmatorio.

Para la realización del tratamiento y el análisis de los datos recogidos, se utilizó el programa estadístico IBM SPSS (versión 22 para Windows). A partir de las 2054 respuestas obtenidas, presentamos a continuación los datos más relevantes (mostrados en porcentajes) de mayor relevancia teniendo en cuenta los estadísticos de media y desviación típica obtenidos con relación a las dimensiones del cuestionario.

En la dimensión autopercepción, la cual incluye aspectos relacionados con la motivación y la percepción de esfuerzo, es significativo que el $77 \%$ de los estudiantes indica que el recurso más valioso en relación con su motivación para aprender son las clases presenciales. Además, un $63 \%$ señala que prefiere leer documentación impresa a leer información digital en línea. Y casi la mitad de los estudiantes (45\%) no está interesada en acudir a charlas o conferencias complementarias a las clases regladas. En relación con las tareas académicas vinculadas a la enseñanza reglada, señalan de forma amplia (85\%) que es muy importante tener claros los objetivos de la tarea para comprender qué se espera de ellos y valorar el esfuerzo a emplear. En otras palabras, parecen más motivados a aprender cuando las tareas reducen la incertidumbre del producto final que se pide. Este dato es interesante por sus implicaciones no solamente en la enseñanza presencial, sino también en modelos virtuales de enseñanza.

Dentro de la dimensión gestión del aprendizaje, se incluyeron ítems relacionados con las categorías de planificación del proceso de aprendizaje, pensamiento crítico, flexibilidad, atención y resolución de problemas. Destaca que un 19\% del alumnado declara utilizar herramientas tecnológicas para gestionar su proceso de trabajo, por lo que el $81 \%$ no las utiliza. En esta categoría de herramientas para la gestión de los aprendizajes hemos incluido calendarios en línea, herramientas de gestión del tiempo, herramientas de gestión del conocimiento y herramientas colaborativas. Coincidiendo con el dato mostrado en la categoría anterior, 
de nuevo aquí aparece la predilección por los estudios en modalidad presencial, pero en un menor porcentaje ( $51 \%$ ). Conocen modalidades de formación en línea como los MOOC, pero en general no muestran interés por ellos. Acerca de la información digital valoran la claridad de la información (45\%) y también los aspectos estéticos de diseño en las presentaciones $(32 \%)$.

Otra de las dimensiones del estudio es la gestión de la información, en la que se les preguntó por las estrategias de búsqueda, gestión, organización, recuperación y creación de información; procesamiento de la información; y sobre temas relacionados con la ética en el uso de recursos. En cuanto a las búsquedas, el $98 \%$ de los estudiantes encuestados declara buscar información en Internet. No obstante, es interesante destacar que el $60 \%$ solamente utiliza motores de búsqueda generalistas (Google) y no acuden a otras herramientas, como pueden ser bases de datos especializadas, ni usan ningún otro tipo de estrategia de búsqueda de información. Además, declaran que la Wikipedia (55\%) es su fuente de información principal en Internet. Un aspecto muy destacado dentro de esta dimensión es el hecho de que casi una cuarta parte indica que no publica ninguna información en red.

Y sobre sus preferencias sobre la naturaleza de los contenidos, mayoritariamente los estudiantes prefieren documentos de carácter académico tradicional en lugar de la información hipertextual o multimedia (95\%). Así mismo, prefieren el papel como soporte de información para estudiar y tomar notas, además de que imprimen la información interesante que encuentran en la red (más del 60\%). Incluso en el caso de información audiovisual/multimedia, toman las notas en papel. Otro dato que redunda sobre este perfil de alumno tradicional es su desconfianza hacia el uso la nube como almacén de información digital, alternativa que solamente reconoce usar el $15 \%$ del alumnado encuestado. Prefieren utilizar sus unidades físicas como pueden ser el propio ordenador, discos externos o dispositivos USB. Por último, en relación a esta dimensión cabe añadir que, desde el punto de vista ético, de modo masivo los estudiantes afirman usar las referencias (más del $83 \%$ ) y aseguran que respetan los derechos de autor evitando el plagio (más del 67\%), que aun siendo porcentajes muy elevados, dejan el margen de casi un 30\% de alumnado que no tiene en consideración el posible plagio cuando elabora un documento, lo cual es también un dato preocupante teniendo en cuenta que son estudiantes universitarios de último curso. 
La última dimensión que compone el instrumento es comunicación, que incluye aspectos relativos a la interacción con personas y aspectos relacionados con dinámicas de trabajo en grupo. Un alto porcentaje reconoce un uso frecuente de redes sociales (casi un 80\%) pero ese uso se centra fundamentalmente en el ocio, no se usa para fines académicos. Como herramientas para la colaboración e interacción muestran sus preferencias por las herramientas de mensajería (41\%), correo electrónico $(28 \%)$ y no alcanza el $6 \%$ el total de estudiantes que usan videoconferencia o chat. La herramienta más utilizada por el alumnado para la realización de proyectos en grupo es Google Doc de Google Drive con un $66 \%$. Las herramientas de redes sociales (Twitter, Facebook...) son utilizadas en menor medida, aunque representan un nada despreciable $22 \%$. Cuando preguntamos a los estudiantes por los aspectos que priorizan cuando trabajan en equipo destaca "construir de forma conjunta" un $58 \%$, seguido de "interaccionar con otros" con un 54\% y en último lugar "compartir recursos" con un (49\%). Con respecto a la valoración de aportaciones y críticas que reciben de otros usuarios el $67 \%$ reconoce tener en cuenta dichas aportaciones.

\section{Conclusiones}

La competencia digital del docente y del alumnado es sin duda un tema de plena actualidad y de interés tanto para las instituciones educativas como para los agentes que intervienen en los procesos formativos. Este impulso ha provocado -entre otras cosas- que actualmente exista un creciente interés por investigar el desarrollo de la competencia digital de los docentes y de los estudiantes, provocando un aumento del número de proyectos y publicaciones al respecto.

Podríamos afirmar que investigar en competencia digital está de moda y Grupo de Investigación de Tecnología Educativa (GITE) es uno de los pioneros en abrir esta línea de investigación y consolidarla como de nuestras principales líneas de trabajo. En 2008 iniciamos este camino con el proyecto "Estudio de las competencias y demanda formativa en TIC de los docentes de las universidades bolivianas y dominicanas" financiado por la Agencia Española de Cooperación Internacional y en 2010 continuamos con el proyecto "Competencias TIC para la docencia en la universidad pública española: indicadores y propuestas para 
la definición de buenas prácticas ${ }^{4 \prime}$ financiado por la Secretaría de Estado de Universidades e Investigación. A estos proyectos les han seguido numerosas contribuciones en congresos internacionales y nacionales, artículos en revistas de impacto, tesis doctorales, libros y capítulos de libros.

Los resultados del Proyecto CAPPLE son nuevas piezas clave para seguir avanzando en el análisis del desarrollo de la competencia digital, en este caso de los estudiantes universitarios españoles. Con este artículo hemos mostrado un enfoque de la competencia digital que utiliza el constructo teórico de PLE para analizar y comprender las competencias para el aprendizaje apoyado en tecnologías, en este caso, centrado en el alumnado universitario. Enfoque compartido con Suárez y Serrano (2016) quienes consideran que, entre los retos de la Universidad actual, ha de ponerse el foco en las oportunidades para actuar, pensar y aprender en los espacios virtuales, así como en la relación entre competencia digital y formación permanente, entendiendo el "PLE como indicador de desarrollo profesional y rasgo de la competencia digital" (p. 228).

Nuestros resultados nos permiten aportar evidencias consistentes y conclusiones robustas con relación a las estrategias y herramientas que los futuros profesionales españoles (alumnado de último curso de Grado) dicen utilizar en los procesos de aprendizaje y comunicación. Estas evidencias derivan en propuestas dirigidas a instituciones universitarias para la mejora de los PLE de los estudiantes universitarios (Gutiérrez, López, Serrano y Solano (2017)). La muestra participante no es representativa y no permite establecer inferencias ni desarrollar conclusiones que se puedan generalizar el resto de la población objeto de estudio. Sin embargo, y a pesar de esta limitación, se considera muy relevante por el elevado número de participantes $(n=2054)$, lo cual hace posible la obtención de evidencias consistentes.

Para finalizar, este artículo permite -con datos- también dar un paso más allá del concepto de nativo digital que tanto ha sido utilizado sin haber sido avalado por estudios rigurosos. En nuestro análisis se ha comprobado que la mayoría del alumnado de último curso de grado tiene presencia en la red, siendo para ellos un espacio habitual para comunicarse. Sin embargo, queda mucho por hacer para lograr que los estudiantes universitarios aprovechen adecuadamente este medio

4 Web: http://www.um.es/competenciastic/ 
como espacio de aprendizaje. En este sentido, el alumnado no ha incorporado herramientas telemáticas para tareas vinculadas a los procesos de gestión del conocimiento. De otro lado, los estudiantes sí buscan información en Internet, pero con limitadas estrategias de búsqueda, quedando muy reducida la lista de las principales herramientas utilizadas para el acceso a la información: Google y Wikipedia. Un resultado alarmante es que uno de cada tres estudiantes universitarios encuestados no tiene en consideración el posible plagio cuando elabora un documento.

Muchas son las cuestiones (como por ejemplo el tratamiento crítico de la información, o la búsqueda y selección de información digital) que siguen quedando pendientes de profundización, tanto con futuros estudios como con la puesta en marcha de medidas concretas para mejorar la competencia digital de los estudiantes universitarios y, en consecuencia, las estrategias y herramientas utilizadas para sus procesos de aprendizaje y comunicación.

Y en este camino seguiremos investigando, usando para ello las competencias docentes e investigadoras adquiridas en la cercanía con el profesor Escudero Muñoz, a quien se le dedica este merecido reconocimiento a través de este número de la Revista Educatio Siglo XXI de la Facultad de Educación de la Universidad de Murcia, institución en la cual hemos compartido muchas y gratas experiencias de aprendizaje con él.

\section{Agradecimientos}

El proyecto CAPPLE ha sido financiado por Ministerio de Economía y Competitividad (España) dentro del «Subprograma de proyectos de investigación fundamental no orientada», en el marco del VI Plan Nacional de Investigación Científica, Desarrollo e innovación Tecnológica (EDU2012-33256).

Agradecemos también la colaboración del profesorado universitario en la difusión del cuestionario y a todos los estudiantes que respondieron nuestra encuesta. 
Entornos Personales de Aprendizaje para la comprensión y desarrollo de la Competencia Digital: análisis de los estudiantes universitarios en España

M. ${ }^{a}$ Paz Prendes Espinosa, Isabel María Solano Fernández, José Luis Serrano Sánchez,

Víctor González Calatayud y M. ${ }^{a}$ del Mar Román García

\section{Referencias bibliográficas}

Baydas, O., Kucuk, S., Yilmaz, R. M., Aydemir, M., y Goktas, Y. (2015). Educational technology research trends from 2002 to 2014. Scientometrics, 105(1), 709-725. DOI: 10.1007/s11192-015-1693-4

Cabero, J. (2003). Replanteando la Tecnología Educativa. Comunicar, 21, 23-30.

Cabero, J. (2016). ¿Qué debemos aprender de las pasadas investigaciones en Tecnología Educativa? Revista interuniversitaria de Investigación en Tecnología Educativa (RIITE), 0, 22-33. DOI: 10.6018/riite/2016/256741

Cabero, J. y Barroso, J. (2016). Formación del profesorado en TIC. Una visión del modelo TPACK. Cultura y Educación, 28(3), 647-663. DOI: 10.1080/11356405.2016.1203526

Cabero, J., Marín, V. y Castaño, C.M. (2015). Validación de la aplicación del modelo TPACK para la formación del profesorado en TIC. @TIC, revista d'innovació educativa, (14), 13-22. DOI: 10.7203/attic.14.4001

Carrera, F.X. y Coiduras, J.L. (2012). Identificación de la competencia digital del profesor universitario: un estudio exploratorio en el ámbito de las ciencias sociales. REDU. Revista de Docencia Universitaria, 10(2), 273-298. Recuperado de: http://red-u.net/ redu/index.php/REDU/article/view/383

Comisión Europea (2006). Competencias clave para el aprendizaje permanente. Recomendación 2006/962/CE del Parlamento Europeo y del Consejo, de 18 de diciembre de 2006, sobre las competencias clave para el aprendizaje permanente [Diario Oficial L 394 de 30.12.2006]

De Benito, B. y Salinas, J. (2016). La Investigación Basada en Diseño en Tecnología Educativa. Revista interuniversitaria de Investigación en Tecnología Educativa (RIITE), 0, 44-59. DOI: 10.6018/riite/2016/260631

Durán, M. Gutiérrez, I. y Prendes, M.P. (2016). Análisis conceptual de modelos de competencia digital del profesorado universitario. Revista Latinoamericana de Tecnología Educativa (RELATEC), 15 (1), 97-114.

Escudero, J.M. (1995). Tecnología e innovación educativa. Bordón, 47 (2), 161-175.

Escudero, J.M. (2006). La formación del profesorado y la garantía del derecho a una buena educación para todos. En J.M. Escudero y A. Luis (Coords.). La formación del profesorado y la mejora de la educación para todos: políticas y prácticas (pp. 21-51). Barcelona: Octaedro.

Escudero, J.M. (2014). Contextos, contenidos y procesos de innovación educativa: ¿el Dónde y el cómo de la tecnología educativa? Docencia e investigación, 24, p.13-37.

Ferrari, A. (2013). DIGCOMP: A Framework for Developing and Understanding Digital Competence in Europe. Sevilla: JRC-IPTS. Recuperado de http://publications.jrc. ec.europa.eu/repository/bitstream/JRC83167/lb-na-26035-enn.pdf

García-Valcárcel, A. (2003). Tecnología Educativa. Implicaciones educativas del desarroIlo tecnológico. Madrid: La Muralla

Gutiérrez, I. (2014). Perfil del profesor universitario español en torno a las competencias en tecnologías de la información y la comunicación. Pixel-Bit, Revista de Medios y Educación, 44, 51-65. DOI: 10.12795/pixelbit.2014.i44.04

Gutiérrez, I., López, P., Serrano, J.L. y Solano, I.M. (2017). Estrategias y herramientas para 
Entornos Personales de Aprendizaje para la comprensión y desarrollo de la Competencia Digital: análisis de los estudiantes universitarios en España

M. ${ }^{a}$ Paz Prendes Espinosa, Isabel María Solano Fernández, José luis Serrano Sánchez, Víctor González Calatayud y M. ${ }^{a}$ del Mar Román García

aprender: descripción de los PLE de los estudiantes universitarios españoles y propuestas para la mejora de la respuesta institucional. En M.P. Prendes y M.M. Román (2017), Entornos personales de aprendizaje: una visión actual de cómo aprender con tecnologías (pp. 65-88). Barcelona: Octaedro.

Hernández, R., Fernández-Collado, P. y Baptista, L. (2006). Metodología de la investigación. México: McGraw-Hill.

Hsu, Y.C., Hung, J.L., y Ching, Y.H. (2013). Trends of educational technology research: more than a decade of international research in six SSCl-indexed refereed journals. Educational Technology Research and Development, 61(4), 685-705. DOI: 10.1007/ s11423-013-9290-9.

Krumsvik, R. (2011). Digital competence in Norwegian teacher education and schools. Högre utbildning, 1(1), 39-51. Recuperado de http://journals.lub.lu.se/index.php/hus/ article/view/4578

Ley Orgánica de Educación (LOE) (Ley Orgánica 2/2006, 3 de mayo). Boletín Oficial del Estado, n 106, 2006, 4 mayo.

Ley Orgánica para la mejora de la calidad educativa (LOMCE) (Ley Orgánica 8/2013, 10 de diciembre). Boletín Oficial del Estado, n² 295, 2013, 10 de diciembre.

Marcovitch, J. (2002). La universidad (im) posible. Madrid: Cambridge University Press.

Mishra, P. y Koehler, M. (2006). Technological pedagogical content knowledge: A Framework for Teacher knowledge. Teachers College Record, 108(6), 1017-1054. Recuperado de http://punya.educ.msu.edu/publications/journal_articles/mishra-koehlertcr2006.pdf

Monereo, C. (2005). Internet y competencias básicas. Aprender a colaborar a comunicarse, a participar, a aprender. Barcelona: Graó.

Pavié, A. (2011). Formación docente: hacia una definición del concepto de competencia profesional docente. Revista Electrónica Interuniversitaria de Formación del Profesorado, 14(1), 67-80. Recuperado de http//www.aufop.com

Perrenoud, P. (2004). Diez nuevas competencias para enseñar. Barcelona: Graó.

Prendes, M.P y Serrano, J.L. (2016). En busca de la Tecnología Educativa: La disrupción desde los márgenes. Revista interuniversitaria de Investigación en Tecnología Educativa (RIITE), 0, 6-16. DOI: 10.6018/riite/2016/263771

Prendes, M.P, Castañeda, L., Ovelar, R. y Carrera, X. (2014). Componentes básicos para el análisis de los PLE de los futuros profesionales españoles: En los albores del proyecto CAPPLE. EDUTEC, Revista electrónica de Tecnológica Educativa, 47, 1-15. Recuperado de http://www.edutec.es/revista/index.php/edutec-e/article/viewFile/139/pdf_4

Prendes, M.P, Castañeda, L., Solano, I., Roig, R., Aguiar, M.P., y Serrano, J.L. (2016). Validation of a Questionnaire on Work and Learning Habits for Future Professionals: Exploring Personal Learning Environments. RELIEVE, 22(2). http://doi.org/10.7203/ relieve.22.2.7228

Real Decreto 126/2014, del Currículo básico de la Educación Primaria. Boletín Oficial del Estado, n 52, 2014, 1 de marzo.

Sancho, J.M. (1998). La tecnología: Un modo de transformar el mundo cargado de ambivalencia, en Sancho, J.M. (coord.). Para una Tecnología Educativa (pp. 13-38). Barcelona: Horsori. 
Entornos Personales de Aprendizaje para la comprensión y desarrollo de la Competencia Digital: análisis de los estudiantes universitarios en España

M. ${ }^{a}$ Paz Prendes Espinosa, Isabel María Solano Fernández, José Luis Serrano Sánchez,

Víctor González Calatayud y M. ${ }^{a}$ del Mar Román García

Serrano, J.L., Gutiérrez, I. y Prendes, M.P. (2016). Internet como recurso para enseñar y aprender: Una aproximación práctica a la Tecnología Educativa. Sevilla: Eduforma.

Stake, R. E. (2010). Qualitative Research: Studying How Things Work. New York, NY: Guilford Press.

Suárez, C. y Serrano, J.L. (2016). Competencia digital y construcción de entornos personales de aprendizaje como retos de la formación universitaria. En C. Suárez, D. Marín y D. Palomares. Retos de la educación en tiempos de cambio (pp. 227-252). Tirant Lo Blanch.

Tejada, J. y Navío, A. (2005). El desarrollo y la gestión de competencias profesionales: una mirada desde la formación. Revista Iberoamericana de Educación, 37(2), 1-15. Recuperado de http://www.rieoei.org/deloslectores/1089Tejada.pdf

Valverde, J. (2016). La investigación en Tecnología Educativa y las nuevas ecologías del aprendizaje: Design-Baes Research (DBR). Revista interuniversitaria de Investigación en Tecnología Educativa (RIITE), 0, 60-73. DOI: 10.6018/riite/2016/257931 